\title{
Ultrafast imaging of terahertz electric waveforms using quantum dots
}

\author{
Moritz B. Heindl ${ }^{1}$, Nicholas Kirkwood ${ }^{2}$, Tobias Lauster ${ }^{3}$, Julia A. Lang ${ }^{1}$, Markus Retsch³ ${ }^{3}$ Paul Mulvaney $\mathbb{D}^{2}$ and \\ Georg Herink (iD ${ }^{1 \times}$
}

\begin{abstract}
Microscopic electric fields govern the majority of elementary excitations in condensed matter and drive electronics at frequencies approaching the Terahertz $(\mathrm{THz})$ regime. However, only few imaging schemes are able to resolve subwavelength fields in the THz range, such as scanning-probe techniques, electro-optic sampling, and ultrafast electron microscopy. Still, intrinsic constraints on sample geometry, acquisition speed and field strength limit their applicability. Here, we harness the quantum-confined Stark-effect to encode ultrafast electric near-fields into colloidal quantum dot luminescence. Our approach, termed Quantum-probe Field Microscopy (QFIM), combines far-field imaging of visible photons with phase-resolved sampling of electric waveforms. By capturing ultrafast movies, we spatio-temporally resolve a Terahertz resonance inside a bowtie antenna and unveil the propagation of a Terahertz waveguide excitation deeply in the sub-wavelength regime. The demonstrated QFIM approach is compatible with strong-field excitation and sub-micrometer resolution -introducing a direct route towards ultrafast field imaging of complex nanodevices inoperando.
\end{abstract}

\section{Introduction}

The detection of radiation-including human vision-is typically sensitive to the energy carried by an electromagnetic wave rather than its fields. Heinrich Hertz succeeded to prove the existence of electromagnetic fields by conversion into incoherent visible fluorescence ${ }^{1}$. Today, electric waveforms can coherently be sampled with ultrashort laser pulses $^{2-4}$ to directly access the temporal signatures of charge motion and quasi-particle excitations in condensed matter systems up to the visible spectrum $^{5}$. Yet, relevant field distributions are often confined to microscopic scales significantly below the diffraction limit-arising from inhomogeneity of materials, microstructures or intrinsic confinement of lightmatter excitations ${ }^{6-8}$. Only a few approaches spatially

Correspondence: Georg Herink (georg.herink@uni-bayreuth.de)

${ }^{1}$ Experimental Physics VIII - Ultrafast Dynamics, University of Bayreuth, Bayreuth, Germany

${ }^{2} A R C$ Centre of Excellence in Exciton Science, School of Chemistry, University of Melbourne, Melbourne, Australia

Full list of author information is available at the end of the article resolve local electric near-field waveforms up to multiTerahertz frequencies, including raster-scanned photoconductive switches and electro-optic microscopy ${ }^{9-13}$. Enhanced resolution is provided by scattering near-field optical microscopy $^{14-17}$, THz-driven scanning tunneling microscopy ${ }^{18,19}$ and recently emerging ultrafast electron microscopy ${ }^{20-22}$. THz-induced visible luminescence has been employed for imaging spatial field distributions via temporally cumulated effects of strong local fields ${ }^{23-26}$. Sampling $\mathrm{THz}$ electric waveforms in the time-domain using visible fluorescence appears highly desirable as it bears numerous prospects including the access to nanoscopic scales, 3D geometries, high-speed acquisition, and compatibility with strong local fields inside active and nonlinear-driven devices ${ }^{7,27-30}$.

Here, we demonstrate ultrafast far-field imaging of $\mathrm{THz}$ electric near-fields using fluorescence microscopy. We capture visible photons from local quantum dot probes and acquire stroboscopic movies of electric near-field evolutions. The scheme employs the quantum-confined Stark effect $(\mathrm{QCSE})^{31-33}$, encoding electric near-fields 

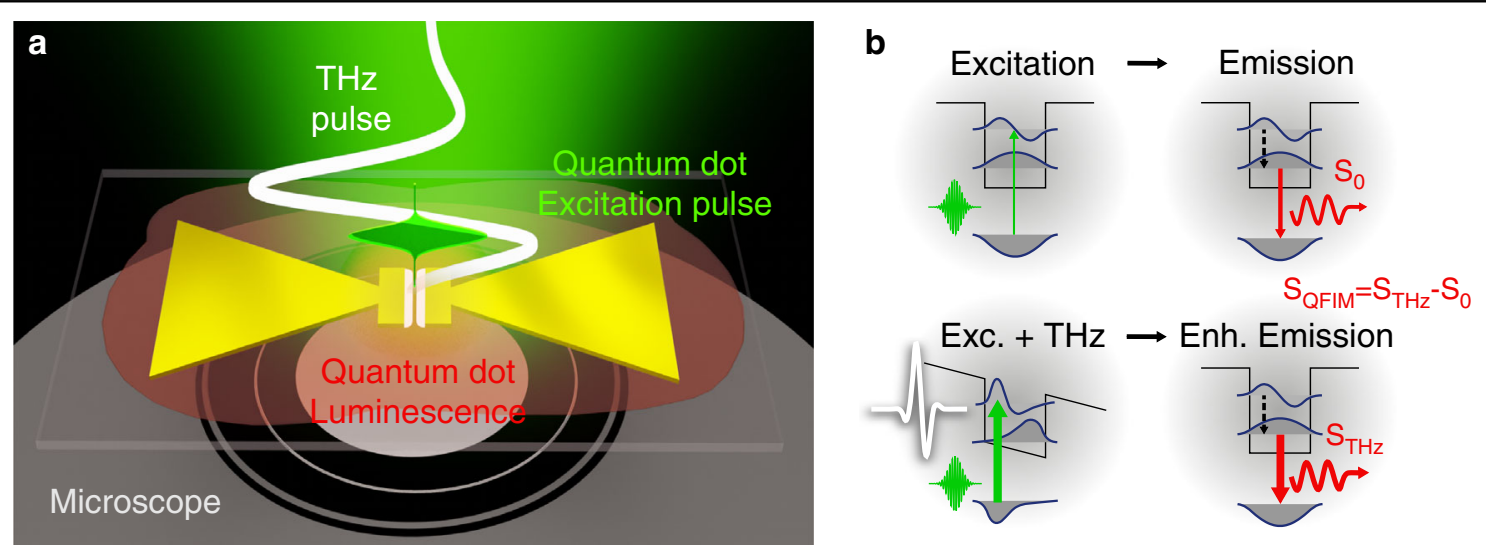

Fig. 1 Quantum-Probe Field Microscopy (QFIM). a Imaging of THz electric near-fields in a fluorescence microscope using quantum dot (QD) luminescence. The absorption of ultrashort visible sampling pulses (green) is modulated via the quantum-confined Stark effect in a layer of nanocrystals (red). b The THz-induced change in the QD band structure can increase the absorption and translates to enhanced luminescence emission, accessible by optical microscopy. The modulated fluorescence yield $S_{\mathrm{QFIM}}=S_{\mathrm{THz}}-S_{0}$ encodes the instantaneous local electric field and snapshot images resolve the spatio-temporally evolution of the near-field waveform

into far-field luminescence modulations via variations of photo-absorption, illustrated in Fig. 1. THz-induced quasi-instantaneous interactions were previously reported for diverse $0 D$-quantum systems ${ }^{26,34,35}$. Harnessing this mechanism, we perform spatially resolved timedomain spectroscopy, and demonstrate the imaging capabilities by resolving the ultrafast electric waveforms of (a) the localized $\mathrm{THz}$ resonance of a bowtie antenna and (b) the propagating $\mathrm{THz}$ gap excitation inside a micro-slit waveguide. Akin to plasmonics in the visible and nearinfrared spectrum, these highly localized excitations arise from collective oscillations of the electron plasma constrained by sub-wavelength geometries.

\section{Results}

Our experiments are based on two-color excitation using single-cycle Terahertz pulses to drive phase-stable near-fields and visible fs-pulses to excite the quantum dot probes, see Fig. 1a. The incident $\mathrm{THz}$ pulses at electric field strengths up to $400 \mathrm{kV} / \mathrm{cm}$ are enhanced in lithographically patterned gold structures. Colloidal CdSe-CdS core-shell nanocrystals, similarly used in voltage sensing applications $^{36,37}$, are deposited as a homogeneous layer of quantum-probes via drop-casting. Luminescence is excited via wide-field illumination in the image plane of a fluorescence microscope with $\sim 150$ fs pulses at wavelengths around $500 \mathrm{~nm}$. We acquire differential images of the emission yield with a CCD camera in the presence and absence of $\mathrm{THz}$ excitation. The difference signal, which we refer to as the QFIM signal $S_{\text {QFIM }}$ in the following, represents the crucial observable for instant local fields.

First, we follow the ultrafast near-field evolution inside a $\mathrm{THz}$ antenna structure, shown in Fig. 2a, with sub-cycle temporal resolution by acquiring a sequence of snapshot images at increasing delays between $\mathrm{THz}$ and visible pulses. Figure $2 \mathrm{~b}$ shows nine exemplary frames out of a series with temporal separation of $\Delta \tau=30 \mathrm{fs}$ (full movie in Media 1). We observe a strong enhancement in the antenna gap and close to the terminal bars ( $\mathrm{THz}$ polarization $\sim 0^{\circ}$ to the antenna axis). The signal is maximized at the edge of each antenna leg and decays symmetrically towards the center of the bowtie as apparent in the snapshot at $\Delta \tau=0$ fs in Fig. 2c, demonstrating a spatial resolution of $\sim 2 \mu \mathrm{m}$ (see Supplementary Information). This pattern visually matches finite-element simulations of the $\mathrm{THz}$ electric near-field, shown in Fig. 2d, and strongly depends on the incident polarization (data for $\mathrm{THz}$ polarization $\sim 90^{\circ}$ to the antenna axis in Supplementary Information). Based on the simulated field enhancement and the incident peak field of $\sim 400 \mathrm{kV} / \mathrm{cm}$, we estimate a maximum near-field strength of $\sim 10 \mathrm{MV} / \mathrm{cm}$.

Analyzing the QFIM signal inside the gap, we demonstrate the extraction of local electric waveforms and characterize the temporal response of the bowtie antenna. As a prerequisite, we study the relation between the maximum field strength $F$ and the peak signal of $S_{\mathrm{QFIM}}$. Measurements with varying incident field strengths yield the dependence $S_{\mathrm{QFIM}} \propto F^{1.9}$ for the quantum dots used in the experiment, as evident in the double-logarithmic representation in Fig. 3b. Thus, the peak signal scales nonlinearly with the maximum incoming field ${ }^{34}$. Employing the rectifying relation and the incident far-field waveform-obtained from calibrated conventional electro-optic sampling (EOS)-, we simulate the local near-field and the resulting QFIM signal using a finiteelement time-domain simulation of the structure and find close agreement with the experimental QFIM trace, see Fig. 3a. The comparison of the incident $\mathrm{THz}$ waveform 


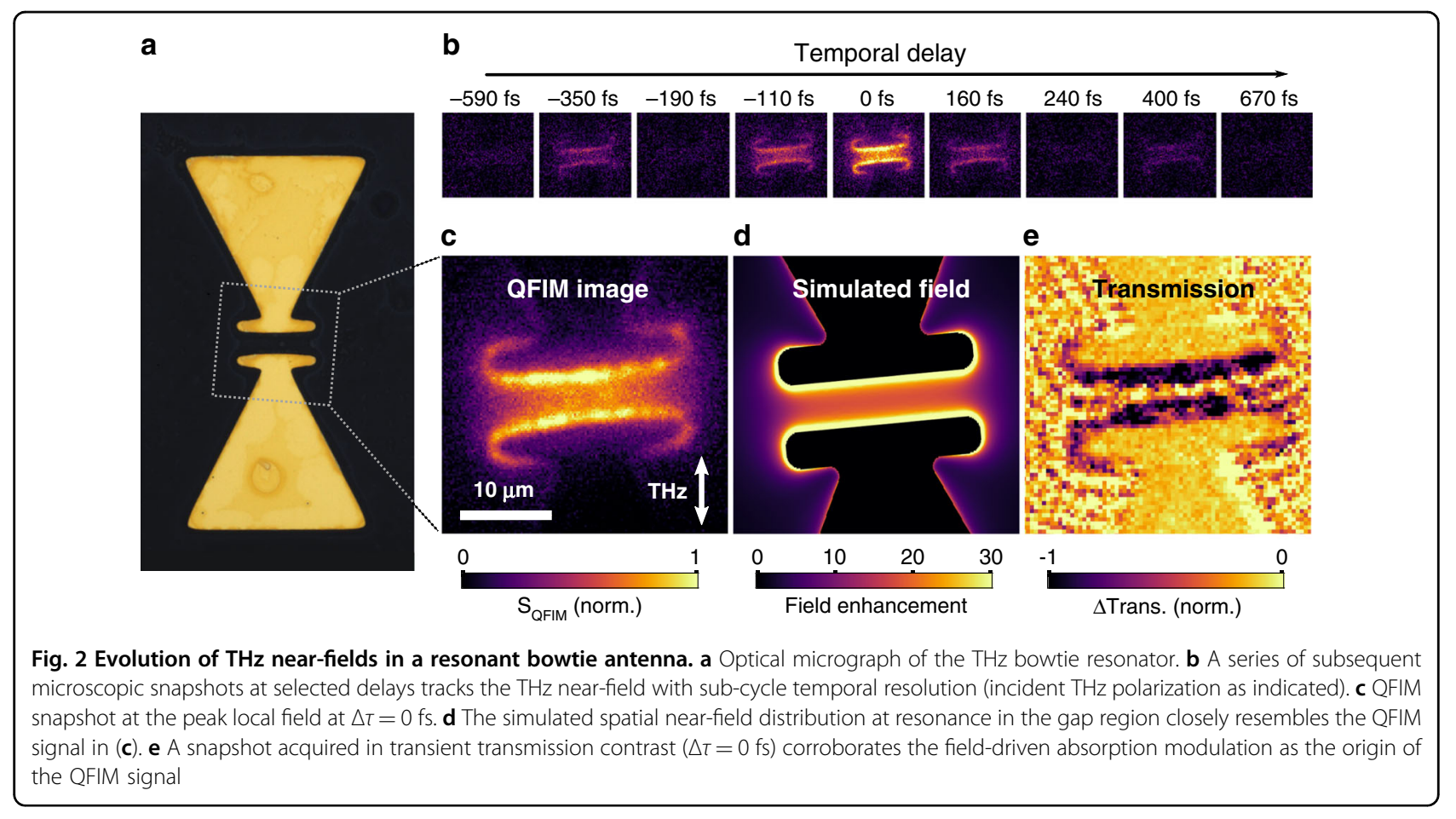

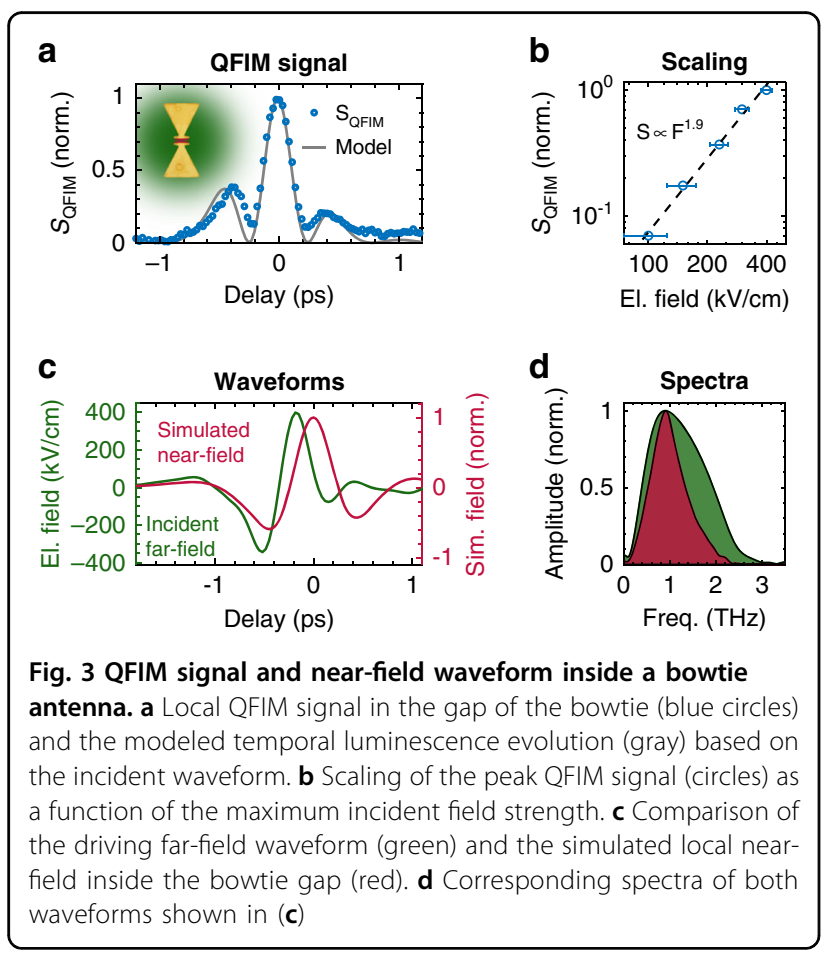

and the simulated near-field evolution is shown in Fig. 3c with corresponding spectra in Fig. 3d. Alternatively, a reconstruction of the near-field in a resonator can be obtained by adapting a single resonance model to the QFIM data, as shown in the Supplementary Information. Depending on the signal quality, direct extraction of near-field waveforms appears feasible via recovery of the polarity and reversal of the nonlinear QFIM signal.

The underlying mechanism enabling the QFIM scheme relies on THz-driven modulations of the electronic band structure in low-dimensional quantum systems ${ }^{31,32}$, i.e., the QCSE in semiconductor nanocrystals ${ }^{33}$. The altered electron and hole wavefunctions induce a quasiinstantaneous change of the optical transition dipole moment. As a result, the photoabsorption may be reduced or enhanced depending on the visible excitation frequency and the accessed electronic states, as previously resolved via transient absorption spectroscopy ${ }^{35}$. We spatially map these changes via luminescence emission microscopy. Specifically, we note that irrespective of much longer luminescence lifetimes $(\sim 10 \mathrm{~ns})$, the temporal sampling resolution is exclusively governed by the ultrafast absorption process. This quasi-instantaneous absorption can alternatively be accessed via transient absorption imaging of the antenna, as shown, e.g., for $\Delta \tau=0$ fs in Fig. 2e, yielding a pattern complementary to the QFIM signal.

Now, we demonstrate the field-resolved tracking of propagating ultrafast $\mathrm{THz}$ excitations using the QFIM scheme. Specifically, we spatio-temporally resolve a $\mathrm{THz}$ wavepacket traveling along the subwavelength slit of a gold waveguide, as depicted in Fig. 4a. We map the temporal evolution of the QFIM signal along the gap in a $2 \mathrm{D}$ representation $(x, \Delta \tau)$ in Fig. $4 \mathrm{~b}$, resolving two distinct features: First, the horizontal lines arise from the direct field enhancement inside the gap extending over the $\mathrm{THz}$ 
a

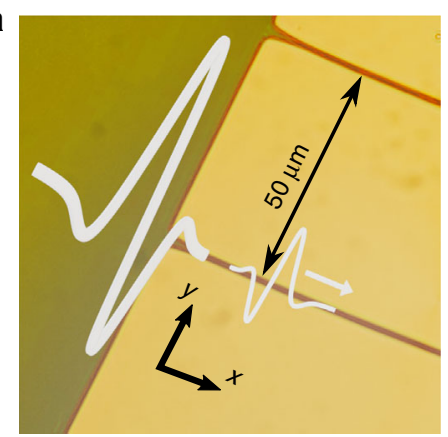

d

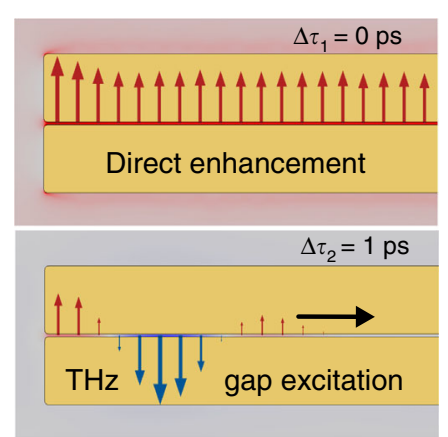

b

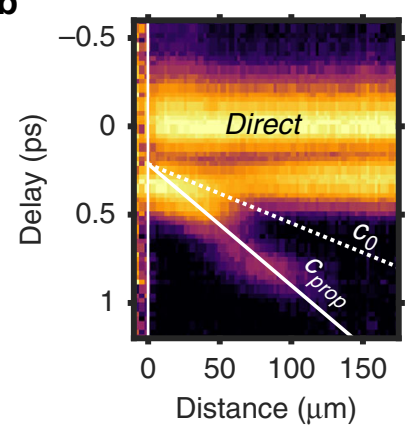

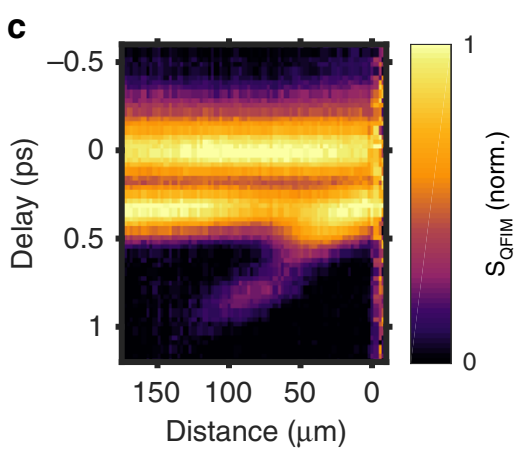

e

Simulated near-field inside gap

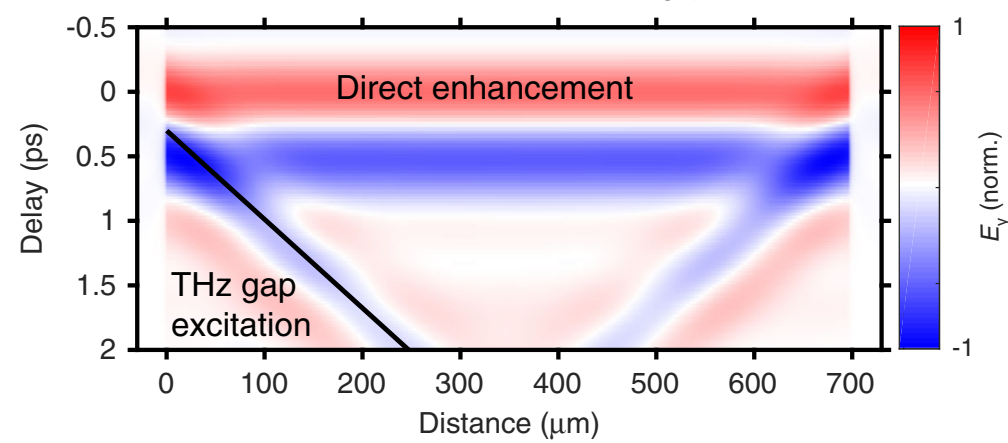

Fig. 4 Temporal imaging of a propagating THz gap excitation. a Launching of a THz waveguide excitation in a micro-slit driven at normal incidence $(2 \mu \mathrm{m}$ gap). $\mathbf{b} 2 \mathrm{D}$ representation $(x, \Delta t)$ of the normalized QFIM waveforms along the slit temporally disentangles the spatially homogeneous direct response of the slit (direct field enhancement) and a THz gap excitation propagating along the waveguide. The peak electric field inside the waveguide propagates with a velocity below $c_{0}$ (dashed line) as quantitatively predicted by time-domain electromagnetic simulation ( $c_{\text {prop, }}$ solid line). c The reversed propagation direction is observed via QFIM for excitation at the opposite side of the waveguide. $\mathbf{d}$ Simulated electric field distributions for two temporal delays illustrate the homogeneous direct enhancement $\left(\Delta \tau_{1}=0 \mathrm{ps}\right)$ and the propagating $\mathrm{THz}$ excitation $\left(\Delta \tau_{2}=1 \mathrm{ps}\right)$. e $2 \mathrm{D}$ representation $(x, \Delta \tau)$ of the simulated electric field evolution along the gap in correspondence to $(\mathbf{b}, \mathbf{c})$

focus. Subsequently, the tilted feature reveals the propagation of a $\mathrm{THz}$ gap excitation with a velocity $c_{\text {prop }}$ below $c_{O}$ emerging from the left edge of the structure. Such propagating plasmonic excitations are confined inside a subwavelength slit and provide the basis for ultrafast circuits-enabling the routing, nanofocusing, and enhancement of infrared radiation ${ }^{12,38-42}$. We corroborate our finding with a time-domain electromagnetic simulation of the ultrafast interaction (see "Materials and methods"), yielding the launching of a $\mathrm{THz}$ wavepacket from the edge with a propagation velocity $c_{\text {prop }}$ (white solid line in Fig. 4b) in agreement with the experimental QFIM dataset. This gap excitation manifests as a spatially oscillating electric field distribution along the slit-in contrast to the unidirectional field of the direct enhancement, illustrated by the simulated fields at two exemplary temporal delays $\left(\Delta \tau_{1}=0 \mathrm{ps}, \Delta \tau_{2}=1 \mathrm{ps}\right)$ in Fig. $4 \mathrm{~d}$. In correspondence to Fig. $4 \mathrm{~b}$, c, we present the simulated electric near-fields as a spatio-temporal map in Fig. 4e. The simulation yields a phase velocity of the waveguide excitation between the vacuum and the substrate of $c_{\text {prop }} \sim c_{0} / 2$. Moreover, we also reproduce the experimentally observed interference of the direct and the propagating pulses. We attribute the different propagation lengths of experiment and simulation to the idealized homogeneous microstructure assumed in the model ${ }^{43}$. Furthermore, the simulation yields a second gap excitation at the opposite side of the $\mathrm{THz}$ waveguide. We experimentally resolve this feature in a QFIM measurement acquired at the right side of the waveguide in Fig. 4c.

\section{Discussion}

We introduce Quantum-probe Field Microscopy to image ultrafast electric near-field waveforms in the timedomain. Our approach utilizes the encoding of momentary $\mathrm{THz}$-fields onto the visible emission of nanocrystals and far-field fluorescence imaging. The underlying $\mathrm{THz}$ fielddriven and quasi-instantaneous QCSE provides a direct link between the luminescence observable and the local electric fields. On this basis, we demonstrate the timeresolved microscopy of near-field waveforms inside a single bowtie antenna-a building block of ultrahighfrequency devices, metamaterials, and strong-field lightmatter interaction experiments ${ }^{27,28}$. Moreover, we observe 
$\mathrm{THz}$ propagation inside a gap deeply in the subwavelength regime and, thus, introduce the ultrafast sampling of propagating electric fields inside confined structures in the time domain. These results motivate the application of QFIM for imaging electric waveforms of surface excitations, including $\mathrm{THz}$ phonon and plasmon polaritons on bulk surfaces and 2D heterostructures $^{44,45}$. In contrast to near-field scattering microscopy based on nanotips, our scheme is compatible with strong driving fields and we envision unprecedented insights to $\mathrm{THz}$-driven nonlinear dynamics, such as interactions between polaritonic wavepackets $^{7,29}$. Finally, we highlight the prospect of QFIM for imaging $\mathrm{THz}$ fields at the nanoscale using optical superresolution microscopy ${ }^{46}$, paving a promising way towards ultrafast nanoscopy of strong electric fields inside nonlinearly driven nanosystems.

\section{Materials and methods Ultrafast QFIM microscope}

We generate high-field single-cycle $\mathrm{THz}$ pulses by the tilted pulse front method ${ }^{47}$ in a $\mathrm{MgO}: \mathrm{LiNbO}_{3}$ crystal using pulses from an amplified $10 \mathrm{kHz}$ Yb-laser system (central wavelength $1030 \mathrm{~nm}$, pulse energy $1 \mathrm{~mJ}$ ), see Fig. S1 in the Supplementary Information. For the quantum dot excitation, we employ laser pulses from an optical parametric amplifier (OPA) at $530 \mathrm{~nm}$ or $480 \mathrm{~nm}$ wavelength, optimized for QFIM signal strength. The vertically polarized $\mathrm{THz}$ beam is focused on the sample with a $90^{\circ}$-off-axis parabolic gold mirror. We obtain a maximum field strength of $400 \mathrm{kV} / \mathrm{cm}$ in the sample plane and a peak frequency of $\sim 0.9 \mathrm{THz}$ via calibrated EO sampling using a $100 \mu \mathrm{m}$ thick $<110>\mathrm{GaP}$ crystal. In addition, the THz field strength can be varied by polarization rotation of the pump pulses used for $\mathrm{THz}$ generation. The OPA beam provides wide-field excitation in the sample plane. Luminescence is collected by a microscope objective. We acquire luminescence images with a cooled CCD camera. The pump pulses used for $\mathrm{THz}$ generation are chopped at a few $\mathrm{Hz}$, and we capture synchronized luminescence images with and without $\mathrm{THz}$ pumping. The consecutive image sequences are digitally subtracted to obtain the $\mathrm{THz}$-induced difference signal. Ultrafast temporal resolution in this pumpprobe scheme is obtained via scanning the temporal delay $\Delta \tau$ between $\mathrm{THz}$ pump pulses and visible excitation pulses via a mechanical delay stage.

\section{Electromagnetic simulations}

We employ a finite element solver (COMSOL Multiphysics) to calculate the electric near-fields of the structures. The model for the bowtie resonator consists of the gold antenna on a soda lime glass substrate ${ }^{48,49}$. For the propagating $\mathrm{THz}$ waveguide excitation, we employ a model consisting of two conducting metal bars (periodicity $50 \mu \mathrm{m}$, length $700 \mu \mathrm{m}$, gap $2 \mu \mathrm{m}$ ) on a soda lime glass substrate. We excite the structures using a plane wave single-cycle $\mathrm{THz}$ pulse (polarization perpendicular to the gap, center frequency $0.9 \mathrm{THz}$ ).

Details on the fabrication of gold microstructures, the synthesis of $\mathrm{CdSe}-\mathrm{CdS}$ quantum dots and the polarization dependence of the bowtie antenna are presented in the Supplementary Information.

\section{Acknowledgements \\ We thank J. Koehler and M. Lippitz for experimental equipment and valuable discussions. \\ Author details \\ 'Experimental Physics VIII - Ultrafast Dynamics, University of Bayreuth, Bayreuth, Germany. ${ }^{2}$ ARC Centre of Excellence in Exciton Science, School of Chemistry, University of Melbourne, Melbourne, Australia. ${ }^{3}$ Physical Chemistry I, University of Bayreuth, Bayreuth, Germany}

\section{Author contributions}

M.B.H. and G.H. conceived the experiment. N.K. synthesized and characterized the quantum dots. T.L. fabricated the microstructures. J.A.L. and M.B.H. performed numerical near-field simulations. M.B.H. recorded the QFIM data. M.B.H. and G.H. analyzed the data and drafted the manuscript. All authors contributed to the interpretation of the data and the writing of the final manuscript

\section{Funding}

This work was funded by the Deutsche Forschungsgemeinschaft (DFG, German Research Foundation) via project 403711541. T.L. acknowledges funding from the European Research Council (ERC) under the European Union's Horizon 2020 research program (grant agreement no. 714968). N.K. and P.M. thank the ARC for support through grant CE170100026. Open Access funding enabled and organized by Projekt DEAL.

\section{Conflict of interest}

The authors declare no competing interests.

Supplementary information The online version contains supplementary material available at https://doi.org/10.1038/s41377-021-00693-5.

Received: 23 September 2021 Revised: 26 November 2021 Accepted: 1 December 2021

Published online: 01 January 2022

\section{References}

1. Hertz, H. Ueber electrodynamische Wellen im Luftraume und deren Reflexion. Ann. Phys. 270, 609-623 (1888).

2. Wu, Q. \& Zhang, X. -C. Free-space electro-optic sampling of terahertz beams. Appl. Phys. Lett. 67, 3523-3525 (1995).

3. Leitenstorfer, A., Hunsche, S., Shah, J., Nuss, M. C. \& Knox, W. H. Detectors and sources for ultrabroadband electro-optic sampling: experiment and theory. Appl. Phys. Lett. 74, 1516-1518 (1999).

4. Keiber, S. et al. Electro-optic sampling of near-infrared waveforms. Nat. Photonics 10, 159-162 (2016).

5. Goulielmakis, E. et al. Direct measurement of light waves. Science $\mathbf{3 0 5}$ 1267-1269 (2004).

6. Huber, A. J., Keilmann, F., Wittborn, J., Aizpurua, J. \& Hillenbrand, R. Terahertz near-field nanoscopy of mobile carriers in single semiconductor nanodevices. Nano Lett. 8, 3766-3770 (2008).

7. Basov, D. N., Fogler, M. M. \& Abajo, F. J. Polaritons in van der Waals materials. Science 354, aag1992 (2016).

8. Lundeberg, M. B. et al. Tuning quantum nonlocal effects in graphene plasmonics. Science 357, 187-191 (2017).

9. Jepsen, P. U., Jacobsen, R. H. \& Keiding, S. R. Generation and detection of terahertz pulses from biased semiconductor antennas. JOSA B 13, 2424-2436 (1996). 
10. Hunsche, S., Koch, M., Brener, I. \& Nuss, M. C. THz near-field imaging. Opt. Commun. 150, 22-26 (1998).

11. Feurer, T., Vaughan, J. C. \& Nelson, K. A. Spatiotemporal coherent control of lattice vibrational waves. Science 299, 374-377 (2003).

12. Wächter, M., Nagel, M. \& Kurz, H. Metallic slit waveguide for dispersion-free low-loss terahertz signal transmission. Appl. Phys. Lett. 90, 061111 (2007).

13. Blanchard, F. et al. Real-time terahertz near-field microscope. Opt. Express 19, 8277 (2011).

14. Knoll, B. \& Keilmann, F. Near-field probing of vibrational absorption for chemical microscopy. Nature 399, 134-137 (1999).

15. Chen, H.-T., Kersting, R. \& Cho, G. C. Terahertz imaging with nanometer resolution. Appl. Phys. Lett. 83, 3009-3011 (2003).

16. Ribbeck, H.-Gvon et al. Spectroscopic THz near-field microscope. Opt. Express 16, 3430-3438 (2008).

17. Eisele, M. et al. Ultrafast multi-terahertz nano-spectroscopy with sub-cycle temporal resolution. Nat. Photonics 8, 841-845 (2014).

18. Cocker, T. L. et al. An ultrafast terahertz scanning tunnelling microscope. Nat. Photonics 7, 620-625 (2013).

19. Peller, D. et al. Quantitative sampling of atomic-scale electromagnetic waveforms. Nat. Photonics 15, 143-147 (2021).

20. Zewail, A. H. Four-dimensional electron microscopy. Science 328, 187-193 (2010).

21. Ryabov, A. \& Baum, P. Electron microscopy of electromagnetic waveforms. Science 353, 374-377 (2016).

22. Feist, A. et al. Ultrafast transmission electron microscopy using a laser-driven field emitter: femtosecond resolution with a high coherence electron beam. Ultramicroscopy 176, 63-73 (2017).

23. Hirori, $\mathrm{H}$. et al. Extraordinary carrier multiplication gated by a picosecond electric field pulse. Nat. Commun. 2, 594 (2011).

24. Lange, $C$. et al. Extremely nonperturbative nonlinearities in GaAs driven by atomically strong terahertz fields in gold metamaterials. Phys. Rev. Lett. 113, 227401 (2014).

25. Iwaszczuk, K., Zalkovskij, M., Strikwerda, A. C. \& Jepsen, P. U. Nitrogen plasma formation through terahertz-induced ultrafast electron field emission. Optica 2, 116 (2015)

26. Pein, B. C. et al. Terahertz-driven luminescence and colossal stark effect in CdSe-CdS colloidal quantum dots. Nano Lett. 17, 5375-5380 (2017).

27. Chen, H.-T. et al. Active terahertz metamaterial devices. Nature 444, 597-600 (2006).

28. Lin, Y.-M. et al. 100-GHz transistors from wafer-scale epitaxial graphene. Science 327, 662-662 (2010).

29. Kauranen, M. \& Zayats, A. V. Nonlinear plasmonics. Nat. Photonics 6, 737-748 (2012).

30. Hentschel, M., Schäferling, M., Duan, X., Giessen, H. \& Liu, N. Chiral plasmonics. Sci. Adv. 3, e1602735 (2017).
31. Mendez, E. E. et al. Effect of an electric field on the luminescence of GaAs quantum wells. Phys. Rev. B 26, 7101-7104 (1982)

32. Miller, D. A. B. et al. Band-edge electroabsorption in quantum well structures: the quantum-confined Stark effect. Phys. Rev. Lett. 53, 2173-2176 (1984)

33. Empedocles, S. A. \& Bawendi, M. G. Quantum-confined Stark effect in single CdSe nanocrystallite quantum dots. Science 278, 2114-2117 (1997).

34. Hoffmann, M. C., Monozon, B. S., Livshits, D., Rafailov, E. U. \& Turchinovich, D. Terahertz electro-absorption effect enabling femtosecond all-optical switching in semiconductor quantum dots. Appl. Phys. Lett. 97, 231108 (2010).

35. Pein, B. C. et al. Terahertz-driven stark spectroscopy of CdSe and CdSe-CdS core-shell quantum dots. Nano Lett. 19, 8125-8131 (2019).

36. Park, K., Deutsch, Z., Li, J. J., Oron, D. \& Weiss, S. Single molecule quantumconfined Stark effect measurements of semiconductor nanoparticles at room temperature. ACS Nano 6, 10013-10023 (2012).

37. Kuo, Y. et al. Characterizing the quantum-confined Stark effect in semiconductor quantum dots and nanorods for single-molecule electrophysiology. ACS Photonics 5, 4788-4800 (2018).

38. Grischkowsky, D. R. Optoelectronic characterization of transmission lines and waveguides by terahertz time-domain spectroscopy. IEEE J. Sel. Top. Quantum Electron. 6, 1122-1135 (2000).

39. Bozhevolnyi, S. I., Volkov, V. S., Devaux, E., Laluet, J.-Y. \& Ebbesen, T. W. Channel plasmon subwavelength waveguide components including interferometers and ring resonators. Nature 440, 508-511 (2006).

40. Schnell, M. et al. Nanofocusing of mid-infrared energy with tapered transmission lines. Nat. Photonics 5, 283-287 (2011).

41. Chen, Y. et al. Efficient interfacing photonic and long-range dielectric-loaded plasmonic waveguides. Opt. Express 23, 9100 (2015).

42. Prämassing, M., Liebtrau, M., Schill, H. J., Irsen, S. \& Linden, S. Interferometric near-field characterization of plasmonic slot waveguides in single- and polycrystalline gold films. Opt. Express 28, 12998 (2020).

43. Kuttge, M. et al. Loss mechanisms of surface plasmon polaritons on gold probed by cathodoluminescence imaging spectroscopy. Appl. Phys. Lett. 93 113110 (2008).

44. Alonso-González, P. et al. Controlling graphene plasmons with resonant meta antennas and spatial conductivity patterns. Science 344, 1369-1373 (2014).

45. Seren, H. R. et al. Nonlinear terahertz devices utilizing semiconducting plasmonic metamaterials. Light Sci. Appl. 5, e16078-e16078 (2016).

46. Hanne, J. et al. STED nanoscopy with fluorescent quantum dots. Nat. Commun. 6, 7127 (2015).

47. Hebling, J., Almasi, G., Kozma, I. \& Kuhl, J. Velocity matching by pulse front tilting for large area THz-pulse generation. Opt. Express 10, 1161 (2002).

48. Johnson, P. B. \& Christy, R. W. Optical constants of the noble metals. Phys. Rev. B 6, 4370-4379 (1972).

49. Rubin, M. Optical properties of soda lime silica glasses. Sol. Energy Mater. 12 275-288 (1985). 